\title{
Spin effects in hadronic top quark pair production
}

\author{
Werner Bernreuther* \\ Institut für Theoretische Physik, RWTH Aachen, 52056 Aachen, Germany \\ E-mail: breutherephysik.rwth-aachen.de
}

\section{Michael Fücker}

Institut für Theoretische Physik, RWTH Aachen, 52056 Aachen, Germany

E-mail: fuecker@physik.rwth-aachen.de

\section{Zong-Guo Si}

Department of Physics, Shandong University, Jinan, Shandong 250100, China

E-mail: zgsiesdu.edu.cn

We consider hadronic $t \bar{t}$ production and decay and review results about $t \bar{t}$ spin correlations and top quark polarization at NLO in the QCD and weak gauge couplings. Moreover, we discuss some effects of non-standard heavy Higgs bosons that strongly couple to top quarks.

International Workshop on Top Quark Physics

January 12-15, 2006

Coimbra, Portugal

${ }^{*}$ Speaker. 


\section{Introduction}

The top quark, the heaviest known fundamental particle, is unique in that it offers the possibility to explore the interactions of a bare quark at energies of a few hundred $\mathrm{GeV}$. Information about this particle has been obtained at the Tevatron in recent years (c.f., for instance [1] for a review). The dynamics of top quark production and decay is, however, still not known very precisely. Hopefully this will change in the years to come. There are exciting physics topics to be explored - let us mention only a few of them. In view of its large mass the top quark is an excellent probe of the mechanism that breaks the electroweak gauge symmetry and should therefore play a key role in clarifying the nature of the force(s)/particle(s) responsible for this phenomenon. The top quark is as heavy as a gold atom, yet it behaves as a pointlike particle, according to present knowledge. Does this pointlike behaviour continue once the $t$ quark can be probed at distance scales significantly below $10^{-16} \mathrm{~cm}$ ? Are there new top-quark decay modes, for instance to supersymmetric particles? So far, experimental data are consistent with the Standard Model (SM) prediction that $t \rightarrow W^{+} b$ is the dominant mode - but its branching ratio and the structure of the $t b W$ vertex is not yet known with high accuracy. These and other topics are being and will be addressed at the Tevatron and at the LHC. Needless to say, present and future experimental analyses require precise SM predictions. It is an important asset for top quark physics that the strong (and electroweak) interactions of top quarks can, in most situations, be reliably predicted.

The top quark is an extremely elusive object. Because its mass is so huge, $m_{t}=172.7 \pm 2.9 \mathrm{GeV}$ [2], it can decay into on-shell $W$ bosons. According to the SM it decays into $W+b$ almost 100 percent of the time, and its total decay width is predicted to be $\Gamma_{t} \simeq 1.4 \mathrm{GeV}$. Thus its lifetime $\tau_{t} \simeq 4 \times 10^{-25} \mathrm{~s}$ is significantly smaller than the typical hadronization time $\tau_{\text {had }} \sim 1 / \Lambda_{Q C D}$. Once produced, $t$ and/or $\bar{t}$ quarks will have decayed before they can form hadronic bound states $(t \bar{q})$, $\left(t q q^{\prime}\right)$. Fragmentation effects are thus expected to play no role in the time span between the production and decay of top quarks. This has important consequences, in particular for top quark spin phenomena. In single top or in $t \bar{t}$ pair production, these quarks will be produced in a specific spin configuration which is due to the production dynamics. Because hadronization does not take place this spin information is transfered to the $t$ and $\bar{t}$ decay products and generates characteristic angular distributions/correlations. The polarization of $t$ and $\bar{t}$ quarks and $t \bar{t}$ spin correlations are "good" observables in the sense that (i) they are reliably calculable and (ii) they are measurable, and are thus well suited to experimentally check predictions of the SM or its extensions. This feature of top quark physics is reminiscent of $\tau$ lepton physics, where spin phenomena were instrumental in the exploration of the interactions of this lepton (for a review, c.f. [3]).

This talk addresses top-spin phenomena in hadronic top quark pair production. (For top-spin issues in single top production, see e.g. [4].) First, some features of semi- and non-leptonic top quark decay will be reviewed. Then we discuss what is known, for $t \bar{t}$ production at the Tevatron and LHC, about top-spin phenomena at next-to-leading order (NLO) in the SM gauge couplings. In particular, we review NLO QCD predictions of $t \bar{t}$ spin correlations and their effects in angular distributions for the dilepton and lepton + jets channels. Finally we discuss, as an example for spin effects caused by interactions beyond the SM, non-standard heavy Higgs bosons that strongly couple to $t \bar{t}$ pairs. 


\section{Semi- and non-leptonic top quark decays}

The semi- and non-leptonic decays of top quarks, $t \rightarrow b+\ell+v_{\ell}, b+q+\bar{q}^{\prime}, q \bar{q}^{\prime}=u \bar{d}, c \bar{s}$, are the dominant decay modes in the SM. An ensemble of top quarks self-analyzes its spin polarization via its weak decays. It is well-known that the charged leptons $\ell=e, \mu, \tau$ are the best analyzers of the top spin. In the following we discuss, for brevity, only the distributions (2.1) below, which are an important ingredient into the predictions outlined in the next section. Consider polarized top decay $t \rightarrow f+\cdots$ in the top quark rest frame. Information about the top spin vector is encoded in the distribution of $\cos \theta_{f}$, where $\theta_{f}$ is the angle between the direction of the particle/jet $f$, used as $t$ spin analyzer, and the polarization vector of the top quark. It is given by

$$
\frac{1}{\Gamma_{f}} \frac{d \Gamma_{f}}{d \cos \theta_{f}}=\frac{1}{2}\left(1+p c_{f} \cos \theta_{f}\right),
$$

where $\Gamma_{f}$ denotes the partial decay width, $p$ is the polarization degree of the ensemble, and $c_{f}$ is the $t$ spin-analyzing power of $f$. In the SM $c_{\ell}=1$ at tree level, while $c_{b}=-c_{W}=0.41$ (assuming reconstruction of the direction of flight of the $W$ boson). That is, for an ensemble of $100 \%$ polarized top quarks, the probability for the $\ell^{+}$being emitted in the direction of the $t$ spin is maximal, while it is zero for the emission opposite to the $t$ spin.

Taking the order $\alpha_{s}$ QCD corrections into account the spin-analyzing power of $f$ decreases slightly due to gluon radiation. To order $\alpha_{s}$ the CKM allowed final states are (i) $\ell v_{\ell}+\mathrm{b}$ jet and $\ell v_{\ell}+\mathrm{b}$ jet + gluon jet in semileptonic decays and (ii) a b jet plus two or three non-b jets in non-leptonic decays. The spin-analyzer quality factors were computed to order $\alpha_{s}$ for the semi- and non-leptonic

\begin{tabular}{ccccccc} 
& $\ell^{+}$ & $\bar{d}$ & $u$ & $b$ & $j_{<}$ & $j_{>}$ \\
\hline LO: & 1 & 1 & -0.32 & -0.41 & 0.51 & 0.2 \\
NLO: & 0.999 & 0.966 & -0.31 & -0.39 & 0.47 &
\end{tabular}

Table 1: Spin-analyzing power $c_{f}$ to LO and NLO in $\alpha_{s}$ for semileptonic [5] and non-leptonic [6] top quark decays.

channels in [5] and in [6], respectively, and are collected in Table 1. For tree-level results, c.f., for instance [7]. For the non-leptonic channels, $j_{<}$and $j_{>}$denote the least energetic and most energetic non-b jet defined by the Durham clustering algorithm. As the identification of the flavors of the quark jets from $W$ decay is not possible - or inefficient in the case of the $c \bar{s}$ final state -, Table 1 shows that, in the case of non-leptonic decays, the least energetic non-b jet is the most efficient top-spin analyzer. This is a consequence of $V-A$ and angular momentum conservation.

The analogous angular distributions for the decays of antitop quarks, $\bar{t} \rightarrow \bar{f}+\cdots$, are proportional to $\left(1-p c_{f} \cos \theta_{f}\right)$, assuming $\mathrm{CP}$ invariance. Violation of this relation requires that the respective decay amplitude has a CP-violating absorptive part [8]. Within the SM such an effect is negliglibly small. Further, we mention that distributions which are more exclusive than (2.1), for instance energy-angle distributions, are also known to order $\alpha_{s}$.

The presence of new interactions in the decays $t \rightarrow b f_{1} f_{2}$ would change the values of the $c_{f}$ given in Table 1. For example, if the decays were mediated by charged Higgs-boson exchange, $c_{b}=-0.4 \rightarrow c_{b}=1$, neglecting interferences with $W$ exchange. A less dramatic effect would be 
caused by a $V+A$ admixture to the Standard Model $t b W$ charged current, $(V-A)+\kappa(V+A)$. In this case neutrino energy-angle distributions are most sensitive to $\kappa \neq 0[9,10]$. The order $\alpha_{s}$ corrections to these and other decay distributions are also available for $\kappa \neq 0[9,10]$. It is important to take the QCD corrections into account in (future) data analyses, as gluon radiation can mimic a small $V+A$ admixture. Although there are tight indirect constraints on $\kappa$ from the measured branching ratio $B(b \rightarrow s \gamma)$, (c.f., for instance [11, 12]), direct searches in future high statistics data on top quark decays can provide unbiased information on this coupling at the level of a few percent [13]. The present experimental error is substantially larger [14].

\section{Hadronic $t \bar{t}$ production}

At the Tevatron and at the LHC the production of $t \bar{t}$ pairs can be analyzed in the dilepton, lepton + jets, and all jets decay channels:

$$
\mathrm{p} \overline{\mathrm{p}}, \mathrm{pp} \rightarrow \mathrm{t} \overline{\mathrm{t} X} \rightarrow\left\{\begin{array}{c}
2 \ell+\mathrm{n} \geq 2 \text { jets }+\mathrm{P}_{\mathrm{T}}^{\text {miss }}, \\
\ell+\mathrm{n} \geq 4 \text { jets }+\mathrm{P}_{\mathrm{T}}^{\text {miss }}, \\
\mathrm{n} \geq 6 \text { jets. }
\end{array}\right.
$$

In the SM the $t \bar{t}$ pairs are dominantly produced by the strong interactions, and the parton reactions $q \bar{q} \rightarrow t \bar{t}, g g \rightarrow t \bar{t}$ are the main ones at the Tevatron and at the LHC, respectively. Predictions for the total cross sections $\sigma(p p, p \bar{p} \rightarrow t \bar{t} X)$ and for distributions of the $t$ and $\bar{t}$ transverse momenta were made on the basis of the NLO results [15], including resummed soft gluon and threshold logarithms [16]. For these observables the $t, \bar{t}$ spins play no role. Predictions for $t \bar{t}$ production and decay including the $t$ and $\bar{t}$ spin degrees of freedom were made so far to NLO QCD in $\alpha_{s}[17,18]$. Let's consider this in a little more detail. At NLO QCD we have the following $2 \rightarrow 6$ and $2 \rightarrow 7$ parton reactions:

$$
\begin{array}{r}
q \bar{q} \stackrel{t \bar{t}}{\longrightarrow} b+\bar{b}+4 f(+ \text { gluon }), \\
g g \stackrel{t \bar{t}}{\longrightarrow} b+\bar{b}+4 f(+ \text { gluon }), \\
g q(\bar{q}) \stackrel{t \bar{t}}{\longrightarrow} b+\bar{b}+4 f+q(\bar{q}),
\end{array}
$$

where $f=q, \ell, v_{\ell}$. Because $\Gamma_{t} \ll m_{t}$, the $t, \bar{t}$ quarks are narrow resonances. Thus the double pole approximation is appropriate (we consider here top as signal, not as background); i.e., the $\mathrm{S}$ matrix elements of the reactions (3.1) - (3.3) (which can proceed through many intermediate states other than $t \bar{t}$ ) are expanded around their poles in the complex $t, \bar{t}$ energy planes, and only the term $\propto\left(D_{t} D_{\bar{t}}\right)^{-1}$ of each matrix element is kept. $\left(D_{t}=p_{t}^{2}-m_{t}^{2}+i m_{t} \Gamma_{t}.\right)$

In the double pole appoximation the radiative corrections - both the real and virtual ones - can be classified into factorizable and non-factorizable corrections. In Fig. 1 this classification is illustrated for virtual corrections. While in Fig. 1 (left) the radiative corrections are confined to the $t \bar{t}$ production and/or the $t$ and/or $\bar{t}$ decay parts of the amplitude, the gluon exchange depicted in Fig. 1 (right) connects the production and decay parts. This classification applies also to the squared matrix elements $|\mathscr{M}|^{2}$ of real gluon radiation.

The differential cross sections for the above parton reactions (3.1) - (3.3) are to $\mathscr{O}\left(\alpha_{s}^{3}\right)$ :

$$
d \sigma_{i}=d \sigma_{i, B}+d \sigma_{i, f a c t}+d \sigma_{i, n f}
$$



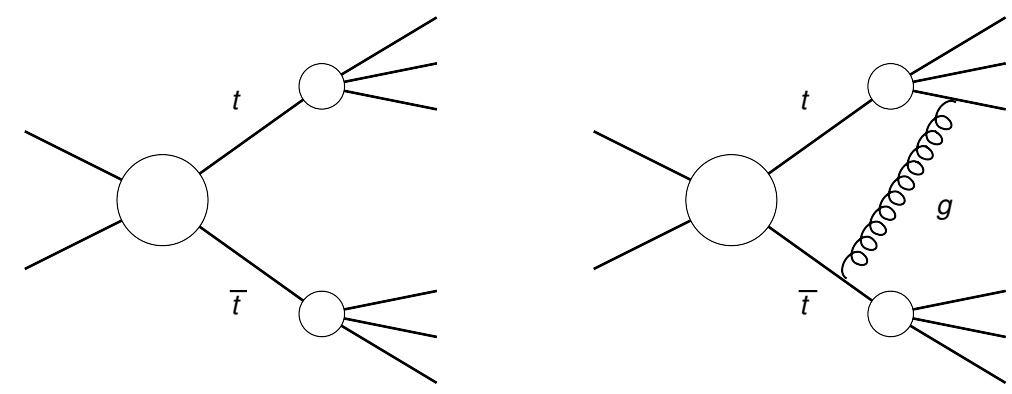

Figure 1: Illustration of factorizable (left) and nonfactorizable (right) virtual QCD corrections.

where $i=q \bar{q}, g g$ denotes the initial state and $d \sigma_{i, B}$ is the lowest order differential cross section. For $i=g q, g \bar{q}$ there is only $d \sigma_{i, B}$ to this order in $\alpha_{s}$. In computing $d \sigma_{i, f a c t}$ we may apply the narrow width approximation ${ }^{1}, \Gamma_{t} / m_{t} \rightarrow 0$, for $t$ and $\bar{t}$. Then

$$
d \sigma_{i, f a c t} \propto \operatorname{Tr}\left(R^{(i)} \rho_{f_{1}} \bar{\rho}_{\bar{f}_{2}}\right)
$$

where $R^{(i)}$ are the $t \bar{t}$ production density matrices and $\rho_{f_{1}}, \bar{\rho}_{\bar{f}_{2}}$ are the density matrices that describe the decays $t \rightarrow f_{1}$ and $\bar{t} \rightarrow \bar{f}_{2}$, respectively. The trace in (3.5) refers to the $t$ and $\bar{t}$ spin labels. The $R^{(i)}$ are known to NLO in $\alpha_{s}$ for all initial states $i=q \bar{q}, g g, g q, g \bar{q}$ and intermediate states $t \bar{t}$ and $t \bar{t} g$ [18]. The NLO decay density matrices can be extracted from the results of $[5,6]$; for details, see [18]. The $R^{(i)}, \rho_{f_{1}}$, and $\bar{\rho}_{\bar{f}_{2}}$ will serve also as building blocks in the computation of $d \sigma_{i, \text { fact }}$ when the intermediate $t, \bar{t}$ are allowed to be also off-shell (in the double pole approximation).

The nonfactorizable order $\alpha_{s}^{3}$ QCD corrections, $d \sigma_{i, n f}$, were computed in [19] for the reactions $q \bar{q}, g g \rightarrow b W^{+} \bar{b} W^{-}$via $t \bar{t}$ intermediate states, and they have recently been recalculated [20]. These corrections are dominated by gluon exchange/radiation with energy $E_{g} \lesssim \mathscr{O}\left(\Gamma_{t}\right)$. They are relevant, e.g., for $t, \bar{t}$ momentum distributions, $t, \bar{t}$ and $t \bar{t}$ invariant mass distributions. However, when computing observables which are inclusive in both the $t$ and $\bar{t}$ invariant masses, the nonfactorizable QCD corrections of order $\alpha_{s}^{3}$ cancel $[21,22,19]$. This result applies also to the angular correlations that will be discussed in the next section.

\section{Top quark polarization and spin correlations}

As emphasized above, top quark polarization and $t \bar{t}$ spin correlations are important tools in exploring the dynamics of these quarks. The SM predicts only a small polarization of $t$ and $\bar{t}$ quarks when pair-produced in hadronic collisions. Strong interactions lead to a polarization of $t$ and $\bar{t}$ quarks orthogonal to the scattering plane, through absorptive parts of the scattering amplitudes of $q \bar{q} \rightarrow t \bar{t}$ and $g g \rightarrow t \bar{t}$, which are of $\mathscr{O}\left(\alpha_{s}^{3}\right)$. This polarization, the size of which is dependent on the parton center-of-mass energy and on the scattering angle, does not exceed $\sim 2 \%$ in magnitude $[23,24]$. Parity-violating weak interactions, which affect both $q \bar{q} \rightarrow t \bar{t}$ and $g g \rightarrow t \bar{t}$, induce a

\footnotetext{
${ }^{1}$ Thus one neglects terms of order $\alpha_{s} \Gamma_{t} / m_{t}$ with respect to the Born term, which are parametrically smaller than the uncalculated NNLO QCD corrections.
} 
top quark polarization in the scattering plane (more general, along some polar vector), which is, however, also small - see below.

On the other hand the correlation of the $t$ and $\bar{t}$ spins in the QCD-induced production reactions is known to be sizeable. In fact, the strength of this correlation depends, like the $t$ and $\bar{t}$ polarization, on the choice of suitable reference axes, which can be interpreted as $t$ and $\bar{t}$ spin quantization axes (i.e. as spin basis) in the approximation of on-shell $t \bar{t}$ production and decay. At the Tevatron the so-called off-diagonal basis [25] and the beam basis [17, 18] yield the strongest correlations, while for the LHC the helicity basis is a good choice - see below. (A procedure to obtain at the LHC a somewhat stronger correlation than in the helicity basis was given in [26].) At the level of the $t \bar{t}$ states their spin correlation with respect to arbitrary reference axes $\hat{\mathbf{a}}, \hat{\mathbf{b}}$ is given by the expectation value:

$$
\mathscr{A}=<4\left(\hat{\mathbf{a}} \cdot \mathbf{s}_{\mathbf{t}}\right)\left(\hat{\mathbf{b}} \cdot \mathbf{s}_{\overline{\mathbf{t}}}\right)>.
$$

This is nothing but the $t \bar{t}$ double spin asymmetry

$$
\mathscr{A}=\frac{\mathrm{N}(\uparrow \uparrow)+\mathrm{N}(\downarrow \downarrow)-\mathrm{N}(\uparrow \downarrow)-\mathrm{N}(\downarrow \uparrow)}{\mathrm{N}(\uparrow \uparrow)+\mathrm{N}(\downarrow \downarrow)+\mathrm{N}(\uparrow \downarrow)+\mathrm{N}(\downarrow \uparrow)}
$$

where the first (second) arrow refers to the $t(\bar{t})$ spin projection onto $\hat{\mathbf{a}}(\hat{\mathbf{b}})$. In the following we choose

$$
\begin{array}{ll}
\hat{\mathbf{a}}=\hat{\mathbf{k}}_{\mathbf{t}}, \quad \hat{\mathbf{b}}=\hat{\mathbf{k}}_{\overline{\mathbf{t}}} & \text { (helicity basis), } \\
\hat{\mathbf{a}}=\hat{\mathbf{b}}=\hat{\mathbf{p}} & \text { (beam basis) } \\
\hat{\mathbf{a}}=\hat{\mathbf{b}}=\hat{\mathbf{d}} & \text { (off-diagonal basis) }
\end{array}
$$

where $\hat{\mathbf{p}}$ denotes the direction of one of the hadron beams (i.e., the $\mathrm{z}$ axis in the laboratory frame), and $\hat{\mathbf{d}}$ is given by

$$
\hat{\mathbf{d}}=\frac{-\hat{\mathbf{p}}+(1-\gamma)\left(\hat{\mathbf{p}} \cdot \hat{\mathbf{k}}_{\mathbf{t}}\right) \hat{\mathbf{k}}_{\mathbf{t}}}{\sqrt{1-\left(\hat{\mathbf{p}} \cdot \hat{\mathbf{k}}_{\mathbf{t}}\right)^{2}\left(1-\gamma^{2}\right)}}, \quad \gamma=E_{t} / m_{t} .
$$

At Born level the vectors involved in these three bases may be defined in the center-of-mass frame of the colliding partons. However, this frame is of no use here, once QCD corrections are taken into account [18]. The reconstruction of this frame requires the measurement of the four-momenta of all final state particles/jets; but for real gluon radiation being collinear to one of the initial partons this is not possible. Thus in this frame the correlation (4.2) is not collinear-safe when using the helicity and the off-diagonal basis. A suitable frame is the zero-momentum frame of the $t \bar{t}$ pair. In the following the three bases above are defined with respect to that frame.

The correlations of the $t, \bar{t}$ spins manifest themselves in decay angular correlations which are to be measured with respect to the chosen reference axes. If the $t(\bar{t})$ decays semileptonically then, as discussed in section 2, the charged lepton is the best spin analyzer, while for non-leptonic $t(\bar{t})$ decays the least-energetic non-b jet will be the best choice, at least from the theoretical point of view. This choice will be made in the following.

Let's now come to predictions at NLO QCD for the Tevatron and the LHC. The dilepton and the lepton + jets channels are best suited for measurements of top-spin effects. Thus we consider

$$
p p, p \bar{p} \longrightarrow t \bar{t} X \rightarrow a b X
$$


where $a, b=\ell^{+}, \ell^{\prime-}, \ell, j_{<}$, and $j_{<}, \ell(\ell=e, \mu)$. To order $\alpha_{s}^{3}$ the $2 \rightarrow 6$ and $2 \rightarrow 7$ parton matrix elements above are involved, and the (differential) cross sections of the reactions (4.4) are given by

$$
\int d \sigma=\sum_{i j} \int d x_{1} d x_{2} f_{i}^{h_{1}}\left(x_{1}, \mu_{F}\right) f_{j}^{h_{2}}\left(x_{2}, \mu_{F}\right)\left[d \Phi_{6}\left(\left|\mathscr{M}_{6, B}\right|^{2}+\delta \mathscr{M}_{6}\right)+d \Phi_{7}\left|\mathscr{M}_{7}\right|^{2}\right],
$$

where $\mu_{F}$ is the factorization scale (which is put, by convention, equal to the renormalization scale), and $d \Phi_{n}$ is the $n$ particle phase space measure. From (4.5) one may obtain, in particular, the following double distribution:

$$
\frac{1}{\sigma} \frac{d^{2} \sigma}{d \cos \theta_{a} d \cos \theta_{b}}=\frac{1}{4}\left[1+B_{1} \cos \theta_{a}+B_{2} \cos \theta_{b}-C \cos \theta_{a} \cos \theta_{b}\right],
$$

where $\theta_{a}\left(\theta_{b}\right)$ is the angle defined between the direction of flight of the particle/jet $a(b)$ in the $t(\bar{t})$ rest frame and the reference axis $\hat{\mathbf{a}}(\hat{\mathbf{b}})$. The structure displayed on the right hand side of (4.6) is obtained when no phase space cuts are applied. The coefficients $B_{1,2}$ reflect the polarization of $t$ and $\bar{t}$ with respect to the axes $\mathbf{a}$ and $\hat{\mathbf{b}}$, respectively. When choosing the beam, off-diagonal, or helicity basis, QCD absorptive parts cannot generate a $t$ and $\bar{t}$ polarization along these axes. Within the SM only weak interaction corrections lead to non-zero coefficients $B_{i}$, which are however small: $\left|B_{1}\right|,\left|B_{2}\right|<1 \%$ (see below). The coefficient $C$ reflects the correlation of the $t$ and $\bar{t}$ spins. Because (4.6) is inclusive in the $t$ and $\bar{t}$ invariant masses, the $\mathscr{O}\left(\alpha_{s}^{3}\right)$ nonfactorizable QCD corrections do not contribute. The following formula holds (in fact it holds, for factorizable corrections, to all orders in $\alpha_{s}$ ) [17]:

$$
C=c_{a} c_{b} \mathscr{A},
$$

where $\mathscr{A}$ is the double spin asymmetry (4.2) and $c_{a}, c_{b}$ are the $t, \bar{t}$ spin-analyzing powers of $a, b$ given in section 2.

Another useful observable for investigating $t \bar{t}$ spin correlations is the opening angle distribution $[27,18]$ :

$$
\frac{1}{\sigma} \frac{d \sigma}{d \cos \varphi}=\frac{1}{2}(1-D \cos \varphi)
$$

where $\varphi=\angle\left(\mathbf{p}_{\mathbf{a}}, \mathbf{p}_{\mathbf{b}}\right)$, with the directions of flight of $a, b$ being defined in the respective $t, \bar{t}$ rest frames. This distribution reflects the correlation of $t$ and $\bar{t}$ spins when projected onto each other, $\left.<\mathbf{s}_{\mathbf{t}} \cdot \mathbf{s}_{\overline{\mathbf{t}}}\right\rangle$.

Table 2 contains the predictions for (4.6) and (4.8) for the dilepton and lepton + jets channels [18]. For the results of Table 2, the CTEQ6L and CTEQ6.1M parton distribution functions [28] were used at LO and NLO, respectively. Moreover, the computations were done in the $\overline{\mathrm{MS}}$ factorization scheme, putting $\mu_{F}=\mu_{R}=m_{t}=175 \mathrm{GeV}$.

The following remarks are in order: (i) The distributions (4.6) and (4.8) were computed for the all-jets channels, too [18]. (ii) Table 2 shows that for the Tevatron the beam basis is practically as good as the off-diagonal basis for detecting the $t \bar{t}$ spin correlations. From the experimental point of view the beam basis is perhaps the best choice. (iii) For the LHC good choices are the double distibution (4.6) in the helicity basis and the opening angle distibution (4.8). The correlation coefficients $C_{h e l}$ and $D$ can be enhanced by cutting away events with large $t \bar{t}$ invariant mass. Based on a Monte Carlo analysis of dilepton and lepton + jets events ref. [29] concludes that these correlations can be measured at the LHC with an error of $\delta D \simeq 4 \%$ and $\delta C_{h e l} \simeq 6 \%$, including systematics. (iv) 


\begin{tabular}{crr|rr}
\multicolumn{3}{c}{ Tevatron, $\sqrt{s}=1.96 \mathrm{TeV}$} & \multicolumn{2}{c}{ LHC, $\sqrt{s}=14 \mathrm{TeV}$} \\
$\ell \ell$ & LO & NLO & \multicolumn{1}{c}{ LO } & \multicolumn{1}{c}{ NLO } \\
\hline $\mathrm{C}_{\text {hel }}$ & -0.471 & -0.352 & 0.319 & 0.326 \\
$\mathrm{C}_{\text {beam }}$ & 0.928 & 0.777 & -0.005 & -0.072 \\
$\mathrm{C}_{\text {off }}$ & 0.937 & 0.782 & -0.027 & -0.089 \\
$D$ & 0.297 & 0.213 & -0.217 & -0.237 \\
\hline$\ell+j$ & & & & \\
\hline $\mathrm{C}_{\text {hel }}$ & -0.240 & -0.168 & 0.163 & 0.158 \\
$\mathrm{C}_{\text {beam }}$ & 0.474 & 0.370 & & \\
$\mathrm{C}_{\text {off }}$ & 0.478 & 0.372 & & \\
$D$ & 0.151 & 0.101 & -0.111 & -0.115 \\
\hline
\end{tabular}

Table 2: Coeffients of angular distributions that reflect $t \bar{t}$ spin correlations, at LO and NLO in $\alpha_{s}$, for the dilepton and lepton + jets channels [18]. For the $\mathrm{LHC} C_{\text {beam }}$ and $C_{\text {off }}$ are not given, as they are very small.

$q \bar{q}$ annihilation and $g g$ fusion contribute with opposite sign to the above distributions. This makes them quite sensitive to the quark and gluon content of the (anti)proton. As it turns out, the NLO predictions given in Table 1 remain basically unchanged when using the PDF set MRST2003 [30] instead of CTEQ6.1, which may not be surprising. (v) The numbers in Table 1 contain no theory errors. Estimating the errors due to PDF uncertainties, scale variations, etc. remains a task for future studies. Furthermore, there are also other distributions of interest for top spin physics, e.g., higher dimensional distributions. For some studies at the Born level, see, e.g. [31].

For a complete discussion of SM effects in hadronic $t \bar{t}$ production (electro)weak corrections should also be taken into account. Interesting questions in this context are: (i) What is the size of the weak corrections at large $t \bar{t}$ invariant mass, say $M_{t \bar{t}}>1 \mathrm{TeV}$ ? The weak corrections must grow in "exclusive" $t \bar{t}$ production (i.e., no real radiation of $W$ and $Z$ bosons) due to the appearance of weakinteraction Sudakov logarithms. (ii) What is the size of the $t$ and $\bar{t}$ polarization or, more general, of parity-violating spin asymmetries induced by the weak interactions? For the investigation of these issues pure QED corrections are of no relevance, and are therefore not considered (beyond the lowest order). At LO, top quark pair production by $q \bar{q}$ annihilation can proceed also by photon and $Z$ boson exchange, $q \bar{q} \rightarrow \gamma^{*}, Z^{*} \rightarrow t \bar{t}$. There is no $\mathscr{O}\left(\alpha \alpha_{s}\right)$ interference term with lowest order gluon exchange because of color mismatch. The one-loop corrections to $d \sigma_{i}$ involving the weak interactions are of order $\alpha \alpha_{s}^{2}$. A large part of these corrections were computed first in [32] (c.f. also [33]). Recently the $\mathscr{O}\left(\alpha \alpha_{s}^{2}\right)$ corrections to $q \bar{q} \rightarrow t \bar{t}$ where completed by the calculation of the infrared-divergent box contributions and of the corresponding real gluon emission diagrams $[34,35]$. For the total $t \bar{t}$ cross sections at the Tevatron and the LHC these electroweak contributions are not important; they are swamped by the QCD uncertainties. However, they are relevant, as compared to the NLO QCD corrections, for the invariant mass distribution $\sigma^{-1} d \sigma / d M_{t \bar{t}}$ or the transverse top momentum distribution $\sigma^{-1} d \sigma / d p_{T}$ at large $M_{t \bar{t}}$ or $p_{T}$, say $M_{t \bar{t}}>1 \mathrm{TeV}$. Thus, these corrections must be taken into account in (future) searches for new physics effects at large $M_{t \bar{t}}$ or $p_{T}$.

The $\mathscr{O}\left(\alpha \alpha_{s}^{2}\right)$ corrections to the $q \bar{q}, g g \rightarrow t \bar{t}$ spin density matrices, calculated in [34, 36], al- 
low to determine the weak-interaction induced top-spin phenomena. As expected these are small effects. As far as the $t \bar{t}$ spin correlations discussed above are concerned, the weak interaction contributions can be neglected. In addition the weak interaction corrections generate P-violating spin effects, in particular a polarization $\left\langle\mathbf{s}_{\mathbf{t}} \cdot \hat{\mathbf{a}}\right\rangle,\left\langle\mathbf{s}_{\overline{\mathbf{t}}} \cdot \hat{\mathbf{b}}\right\rangle$ of the $t$ and $\bar{t}$ quarks along a polar vector, e.g., along the beam direction or along the $t$ and $\bar{t}$ directions of flight. P-violating (single) spin effects are again best analyzed in the $\ell \ell$ and $\ell+j$ channels. If one considers $p p, p \bar{p} \rightarrow t \bar{t} X \rightarrow \ell^{+}+X$, information on the $t$ polarization may be obtained from the angular distributions

$$
\frac{1}{\sigma} \frac{d \sigma}{d \cos \theta_{+}}=\frac{1}{2}\left(1+B \cos \theta_{+}\right)
$$

where $\theta_{+}=\angle\left(\ell^{+}, \hat{\mathbf{a}}\right)$ and $\mathbf{a}$ may be chosen to be the beam axis (Tevatron) or the helicity axis (LHC). In the SM these effects are small, $|B|<1 \%$. The asymmetries resulting from (4.9) may be enhanced somewhat by evaluating them only within appropriately chosen invariant mass bins. In [37] a Pviolating double spin asymmetry was considered (in the helicty basis). Its SM value was found to be small, of order $1 \%$ at the LHC, while larger effects were obtained for some SM extensions (c.f. also [38]). Thus, SM induced P-violating spin effects in $t \bar{t}$ production are too small for being observable at the Tevatron. Very probably this holds also for the LHC. But observables like (4.9) should nevertheless be measured, as they are sensitive tools in the search for new interactions involving top quarks.

\section{Heavy Higgs resonances}

Many phenomenological studies have been made on the use of top spin phenomena, in particular of $t \bar{t}$ spin correlations as tools for searching for new interactions in (hadronic) $t \bar{t}$ production and decay. These studies used either effective Lagrangeans, i.e., anomalous couplings in order to parameterize possible new physics effects $[8,39,40,41,42,43,44,45,46]$, or worked within specific extensions of the SM, including 2-Higgs-doublet models (2HDM) [47, 27, 48, 37, 49], the minimal supersymmetric SM extension (MSSM) [37, 49], and Kaluza-Klein modes [50]. For brevity we consider here only one scenario, namely heavy non-standard Higgs bosons, as predicted by many SM extensions, which strongly couple to top quarks. In the case of 2HDMs or the MSSM the spectrum of physical Higgs particles contains three neutral states: two scalars $h_{1}, h_{2}$ having $J^{P C}=0^{++}$, and a pseudoscalar $A$ with $J^{P C}=0^{-+}$. If the (effective) Higgs potential does not conserve $\mathrm{CP}$, the scalars and the pseudoscalar will mix, and a Higgs state of definite mass will no longer have a definite CP quantum number. Depending on the parameters of the respective model some of these states may be heavy, e.g., $h_{2}$ and $A$, with masses of the order of $300 \mathrm{GeV}$ or larger. Of particular interest here is the case of a pseudoscalar, as $A \rightarrow W^{+} W^{-}, Z Z$ in lowest order. If the ratio of the vacuum expectation values of the two Higgs doublet fields, $\tan \beta$, is of order 1 , these states will strongly couple to top quarks ${ }^{2}$. Consider the production of $\varphi=h_{2}, A$ via gluon fusion at the LHC. These bosons will then preferentially decay into $t \bar{t}$ pairs, $g g \rightarrow \varphi \rightarrow t \bar{t} \longrightarrow$ final state. The amplitude of this reaction interferes with the amplitude of the QCD-induced non-resonant $t \bar{t}$ background, $g g \rightarrow t \bar{t} \rightarrow$ final state, and this interference is not negligible, even in the vicinity of the

\footnotetext{
${ }^{2}$ If $\tan \beta \gg 1$ then heavy Higgs $\rightarrow \tau^{+} \tau^{-}$is an important channel.
} 
resonance, $\sqrt{s} \sim m_{\varphi}$, because the width $\Gamma_{\varphi}$ of the Higgs particle is not expected to be small. The interference generates a peak-dip structure in the $t \bar{t}$ invariant mass distribution $M_{t \bar{t}}$ [51, 27]. Statistically significant signals are possible in the mass range $350 \mathrm{GeV} \lesssim m_{\varphi} \lesssim 500 \mathrm{GeV}$, depending on the strength of the Yukawa couplings and on the width of $\varphi[27,52,53]$. Needless to say, this is a very difficult channel which requires very good $M_{t \bar{t}}$ resolution and a precise knowledge of the SM background contributions to the $M_{t \bar{t}}$ distribution.

Suppose experiments will be lucky and discover a heavy boson in the $t \bar{t}$ channel. The spin of this resonance may be infered from the polar angle distribution of the top quarks. Let's assume the outcome of this analysis is that the resonance has spin zero. How to find out whether it is a scalar or pseudoscalar? In [27] it was proposed to use spin correlations for answering this question and it was found that $\left\langle\mathbf{s}_{\mathbf{t}} \cdot \mathbf{s}_{\overline{\mathbf{t}}}\right\rangle$ is the best choice, which is easy to understand in simple quantum mechanical terms. Consider $g g \rightarrow \varphi \rightarrow t \bar{t}$. If $\varphi$ is a scalar $\left(J^{P C}=0^{++}\right)$then $t \bar{t}$ is in a ${ }^{3} P_{0}$ state, and a simple calculation yields $\left\langle\mathbf{s}_{t} \cdot \mathbf{s}_{\bar{t}}\right\rangle=1 / 4$. If $\varphi$ is a pseudoscalar $\left(J^{P C}=0^{-+}\right)$then $t \bar{t}$ is in a ${ }^{1} S_{0}$ state and $\left\langle\mathbf{s}_{t} \cdot \mathbf{s}_{\bar{t}}\right\rangle=-3 / 4$. However, the striking difference in the values of this correlation is diluted if the non-resonant $t \bar{t}$ background is taken into account. In order to preserve the discriminating power of this spin observable the correlation should be determined only for events with $M_{t \bar{t}}$ in the vicinity of $m_{\varphi}$.

The correlation $\left\langle\mathbf{s}_{\mathbf{t}} \cdot \mathbf{s}_{\overline{\mathbf{t}}}\right\rangle$ induces the opening angle distribution (4.8) which is best studied in the dilepton channel. Depending on the couplings and on the with of $\varphi$ a statistically significant effect may be found with this distribution. It should be determined for $t \bar{t}$ events that lie in a suitably chosen $M_{t \bar{t}}$ bin below $m_{\varphi}$ [27].

If the CP symmetry is broken by the Higgs sector the state $\varphi$ can have both scalar and pseudoscalar couplings to fermions. Then a CP-odd transverse spin-spin correlation and a CP-odd single spin asymmetry is induced in $\varphi \rightarrow f \bar{f}$ [47]. They, in turn, generate corresponding angular correlations and asymmetries [48], for instance in the dilepton and lepton + jets channels.

\section{Conclusions}

Top-quark spin physics remains to be explored, both in single top and in $t \bar{t}$ production and decay. Here we confined ourselves to the discussion of hadronic $t \bar{t}$ production and decay and reviewed results about $t \bar{t}$ spin correlations and $t$ and $\bar{t}$ polarization at NLO in the QCD and weak gauge couplings. Precision measurements of top spin effects at the level of a few percent appear to be feasible at the LHC. Thus spin observables will become important tools in future experimental investigations of the dynamics of top quarks, and this should also motivate further top spin studies within the SM and beyond.

\section{Acknowledgements}

W.B. wishes to thank the workshop organizers for the invitation to this pleasant meeting. This work was supported by the Deutsche Forschungsgemeinschaft (DFG), SFB/TR9, by DFG Graduiertenkolleg of RWTH Aachen, and by the NCET and NSFC of China. 


\section{References}

[1] A. Juste, talk given at this workshop.

[2] The Tevatron Electroweak Working Group, J.F. Arguin et al., "Combination of CDF and D0 results on the top-quark mass," hep-ex/0507091.

[3] A. Stahl, Springer Tracts Mod. Phys. 160 (2000) 1.

[4] C. P. Yuan, talk given at this workshop.

[5] A. Czarnecki, M. Jezabek and J. H. Kühn, Nucl. Phys. B 351 (1991) 70.

[6] A. Brandenburg, Z. G. Si and P. Uwer, Phys. Lett. B 539 (2002) 235 [hep-ph/ 0205023 ].

[7] G. Mahlon and S. J. Parke, Phys. Rev. D 53 (1996) 4886 [hep-ph / 9512264 ].

[8] W. Bernreuther, O. Nachtmann, P. Overmann and T. Schröder, Nucl. Phys. B 388 (1992) 53 [Erratum-ibid. B 406 (1993) 516].

[9] M. Jezabek and J. H. Kühn, Phys. Lett. B 329 (1994) 317.

[10] W. Bernreuther, M. Fücker and Y. Umeda, Phys. Lett. B 582 (2004) 32 [hep-ph / 0308296 ].

[11] F. Larios, M. A. Perez and C. P. Yuan, Phys. Lett. B 457 (1999) 334 [hep-ph/ 9903394 ].

[12] G. Burdman, M. C. Gonzalez-Garcia and S. F. Novaes, Phys. Rev. D 61 (2000) 114016 [hep-ph/9906329].

[13] N. Castro, talk given at this workshop.

[14] E. W. Varnes, talk given at this workshop.

[15] P. Nason, S. Dawson and R. K. Ellis, Nucl. Phys. B 303 (1988) 607; Nucl. Phys. B 327 (1989) 49; W. Beenakker, H. Kuijf, W. L. van Neerven, J. Smith, Phys. Rev. D 40 (1989) 54; W. Beenakker et al., Nucl. Phys. B 351 (1991) 507.

[16] R. Bonciani, S. Catani, M. L. Mangano, P. Nason, Nucl. Phys. B 529 (1998) 424 [hep-ph/9801375].; N. Kidonakis, E. Laenen, S. Moch, R. Vogt, Phys. Rev. D 64 (2001) 114001 [hep-ph/ 01050 41].; N. Kidonakis and R. Vogt, Phys. Rev. D 68 (2003) 114014 [hep-ph/0308222]; M. Cacciari, S. Frixione, M. L. Mangano, P. Nason and G. Ridolfi, JHEP 0404 (2004) 068 [hep-ph / 0303085$].$

[17] W. Bernreuther, A. Brandenburg, Z. G. Si and P. Uwer, Phys. Rev. Lett. 87 (2001) 242002 [hep-ph/0107086].

[18] W. Bernreuther, A. Brandenburg, Z. G. Si and P. Uwer, Nucl. Phys. B 690 (2004) 81 [hep-ph/0403035].

[19] W. Beenakker, F. A. Berends and A. P. Chapovsky, Phys. Lett. B 454 (1999) 129 [hep-ph/9902304].

[20] L. Meyer, Diploma Thesis, RWTH Aachen (2005), unpublished.

[21] V. S. Fadin, V. A. Khoze and A. D. Martin, Phys. Rev. D 49 (1994) 2247.

[22] K. Melnikov and O. I. Yakovlev, Phys. Lett. B 324 (1994) 217.

[23] W. Bernreuther, A. Brandenburg and P. Uwer, Phys. Lett. B 368 (1996) 153 [hep-ph/9510300].

[24] W. G. D. Dharmaratna and G. R. Goldstein, Phys. Rev. D 53 (1996) 1073. 
[25] G. Mahlon and S. Parke, Phys. Lett. B 411 (1997) 173 [hep-ph / 9706304 ].

[26] P. Uwer, Phys. Lett. B 609 (2005) 271 [hep-ph/ 0412097 ].

[27] W. Bernreuther, M. Flesch and P. Haberl, Phys. Rev. D 58 (1998) 114031.

[28] J. Pumplin et al., JHEP 0207 (2002) 012 [hep-ph / 0201195 ].

[29] F. Hubaut, E. Monnier, P. Pralavorio, K. Smolek and V. Simak, Eur. Phys. J. C 44S2 (2005) 13 [hep-ex/0508061].

[30] A. D. Martin, R. G. Roberts, W. J. Stirling and R. S. Thorne, Eur. Phys. J. C 35 (2004) 325 [hep-ph/0308087].

[31] C. A. Nelson et al., Eur. Phys. J. C 45 (2006) 121 [hep-ph / 050624 0]; C. A. Nelson, J. J. Berger and J. R. Wickman, hep-ph/0510348.

[32] W. Beenakker et al., Nucl. Phys. B 411 (1994) 343.

[33] C. Kao, G. A. Ladinsky and C. P. Yuan, Int. J. Mod. Phys. A 12 (1997) 1341.

[34] W. Bernreuther, M. Fücker and Z. G. Si, Phys. Lett. B 633 (2006) 54 [hep-ph / 0508091 ].

[35] J. H. Kühn, A. Scharf and P. Uwer, Eur. Phys. J. C 45 (2006) 139 [hep-ph/ 0508092 ].

[36] W. Bernreuther, M. Fücker and Z. G. Si, to be published.

[37] C. Kao and D. Wackeroth, Phys. Rev. D 61 (2000) 055009 [hep-ph/ 9902202 ].

[38] C. S. Li, C. P. Yuan and H. Y. Zhou, Phys. Lett. B 424 (1998) 76 [arXiv:hep-ph/9709275].

[39] J. P. Ma and A. Brandenburg, Z. Phys. C 56 (1992) 97; A. Brandenburg and J. P. Ma, Phys. Lett. B 298 (1993) 211.

[40] D. Atwood, A. Aeppli and A. Soni, Phys. Rev. Lett. 69 (1992) 2754.

[41] G. L. Kane, G. A. Ladinsky and C. P. Yuan, Phys. Rev. D 45 (1992) 124.

[42] D. Atwood, A. Kagan and T. G. Rizzo, Phys. Rev. D 52 (1995) 6264 [hep-ph/9 407408 ].

[43] P. Haberl, O. Nachtmann and A. Wilch, Phys. Rev. D 53 (1996) 4875 [hep-ph / 9505409 ].

[44] B. Grzadkowski, B. Lampe and K. J. Abraham, Phys. Lett. B 415 (1997) 193 [arXiv:hep-ph/9706489].

[45] K. m. Cheung, Phys. Rev. D 55 (1997) 4430 [hep-ph/9610368].

[46] C. A. Nelson and A. M. Cohen, Eur. Phys. J. C 8 (1999) 393 [arXiv:hep-ph/9806373].

[47] W. Bernreuther and A. Brandenburg, Phys. Lett. B 314 (1993) 104; Phys. Rev. D 49 (1994) 4481 [hep-ph/9312210].

[48] W. Bernreuther, A. Brandenburg and M. Flesch, hep-ph/9812387.

[49] D. Atwood, S. Bar-Shalom, G. Eilam and A. Soni, Phys. Rept. 347 (2001) 1 [arXiv:hep-ph/0006032].

[50] M. Arai, N. Okada, K. Smolek and V. Simak, Phys. Rev. D 70 (2004) 115015 [hep-ph/ 0409273 ].

[51] D. Dicus, A. Stange and S. Willenbrock, Phys. Lett. B 333 (1994) 126.

[52] “ATLAS detector and physics performance. Technical design report. Vol. 2," CERN-LHCC-99-15.

[53] L. Sonnenschein, "The $t \bar{t}$ production in p p collisions at $\sqrt{s}=14 \mathrm{TeV}$ ", PhD Thesis, RWTH Aachen, PITHA 01/04 (2001), unpublished. 